\title{
INDEX TOTIUS HISTORIAE SECUNDUM ORDINEM LIBRORUM ET CAPITULORUM IN EDITIONE PRINCIPE EXHIBITUS.
}

\section{LIBER PRIMUS.}

I. De vita moribusque Bartholomaei archiepiscopi Barensis et magistratibus ante summum pontificatum per eundem gestis . . . . . . . . . . . 7

II. Quae ante novi pontificis electionem ac circa eandem acta sint et quo pacto dictus archiepiscopus electus fuerit . . . . . . . . . . . . . 10

III. De coronatione eiusdem et obedientia illi tanquam vero pontifici exhibita. . . . . . . . . .

IV. De severa atque intempestiva eiusdem initio pontificatus in cardinales atque episcopos obiurgatione

V. De ipsius integritate atque insigni continentia in contemnendis pecuniis . . . . . . . . . .

VI. De Ottonis ducis Brunsvicensis ac Joannae reginae Siciliae uxoris eiusdem in Urbanum studiis . .

VII. De insolentia atque austeritate Urbani deque causis defectionis ab Urbano . . . . . . . . . .

VIII. De his, quae ad extinguenda nascentis seminaria schismatis ab Ottone aliisque gesta sint . . .

IX. Quibus artibus cardinales Galli Italos in suam de alio eligendo pontifice traxerint sententiam, vide illic vaferrimum commentum . . . . . . . 24

X. Clemens VII. antipapa substituitur . . . . . 25

XI. Recessus cardinalium ab Italia in Gallias . . 26

XII. Urbanus novos creans cardinales Neapolitanos quosque indignissimos provehit atque auget honoribus 
XIII. De conflictu militum ,Clementinorum atque RomaPag. norum ad pontem Tiberis [rectius ad pontem Salarium] iuxta urbem atque de caede quorundam civium Romanorum . . . . . . . . . . .

XIV. Caedes, iniuriae atque contumeliae exteris in urbe a Romanis illatae . . . . . . . . . . .

XV. De legatione Urbani ad Carolum Romanorum ac Ludovicum Ungariae reges auxilium orantis. . .

XVI. De insigni Clementis antipape in regios legatos contumelia . . . . . . . . . . . . . . . 32

XVII. Imperator, Hungaria, Polonia, Germania, Italia Urbano vero pontifici obediunt . . . . . . .

XVIII. De morte imperatoris ac Galliarum regis et de suc-

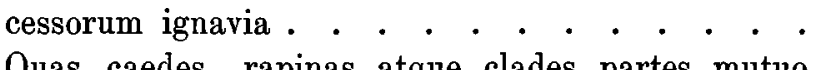

XIX. Quas caedes, rapinas atque clades partes mutuo sibi inflixerint.

XX. Arx sancti Angeli a Clementis praefecto traditur atque a Romanis diruitur. . . . . . . .

XXI. Consilium Urbani de nepote suo extollendo, item de regibus Hungariae atque Siciliae . . . . .

XXII. Sacrae aedes in belli sumptus spoliantur. Carolus dux Siciliae rex declaratur . . . . . . . .

XXIII. Idem Carolus cum proditione Neapolim intrans Ottonem ducem per insidias capit . . . . . .

XXIV. Liberum ac vere Germanum Ottonis responsum. Item bella ab eo in Italia confecta. De Joanna eius uxore hostibus se dedente et de quodam mago

$\mathrm{XXV}$. De miseranda Joannae reginae ad aras iugulatione ac de Margarita uxore Roberti de Orthoiis [rectius Artois]

XXVI. De saevitia atque tyrannide cardinalis legati in Neapolitanos clericos et episcopos . . . . . . 51

XXVII. Ludovicus dux Andegavensis a Clemente Siciliae rex pronunciatus cum exercitu Gallorum Italiam ingreditur. De fide Germanorum militum in eundem

XXVIII. Profectio Urbani ad Neapolim describitur . .

XXIX. Quo pacto Urbanus Campaniam ingressus a Carolo Siciliae rege salutatur . $\cdot \cdot \cdot \cdot c \cdot c c c c c c$

XXX. Auctor in itinere a latronibus caeditur, curandi gratia Neapolim petit . . . . . . . . 
XXXI Urbanus a Carolo Siciliae rege capitur $\cdot{ }^{\circ}$ Pag.

XXXII. Papa rursus liberatur . . . . . . . . . . 62

XXXIII. Incestus ac stuprum Francisci nepotis Urbani . . 63

XXXIV. Franciscus ob flagitium a rege damnatur, per Urbanum rursus reconciliatur . . . . . . . . 64

XXXV. Quae in castris campisque Tarentinis a Ludovico ac Carolo gesta sint. De strage militum Ludovici ob potum fontis venenati . . . . . . . .

XXXVI. Margaritae reginae Siciliae insolentia atque avaritia notatatur . . . . . . . . . . . . . . 67

XXXVII. De metu ac fuga curialium Urbani ad Neapolim ac de pontificis auctorisque ea de re dissertatio

XXXVIII. Arcis Lucerianae pulchritudinem eiusque terrae miram ac raram felicitatem describit auctor. .

XXXIX. De victoria Henrici secundi Romani Imperatoris in Sicilia deque eius illic tropheis. Item de veris atque legitimis Siciliae heredibus scitu dignissima

XL. De amoenitate saltus Luceriani ac Salernitani . . $\quad 75$

XLI. De loco hiemationis Urbanus cum suis consultat . $\quad 76$

XLII. De machinatione atque occultis tractatibus a quibusdam praelatis contra Urbanum . . . . . .

XLIII. De parum verecundis Neapolitanorum ad Urbanum supplicationibus . . . . . . . . . . . .

XLIV. Urbanus archiepiscopos electores imperii galero donans plerosque indignissimos tum creat cardinales

XLV. Sex praestantissimi cardinales Urbano suspecti carceribus durissimis mancipati ab auctore suisque collegis Ĺrbani iussu examinantur . . . . . . .

XLVI. Mare Neapolitanum navigantibus ob piratas infestum, auctor causas disquirit olei ex petris illic stillantis, quae loci eius facies. De Aetna atque Vesuvio montibus . . . . . . . . . . .

XLVII. De saevitia atque avaritia cuiusdam Achillici militis, et quo is pacto aulicos Urbani terra marique insecutus spoliaverit. . . . . . . . . . .

XLVIII. Expostulatio auctoris adversus pontificem, ut sui suorumque rationem habeat orantis . . . . 
XLIX. Auctor Neapolim rediens rursus in latrones incidit, quos, servulis suis male mulctatis atque spoliatis, vix evasit. Item de forma ac moribus Caroli regis

L. Cardinales carceribus educti innocentiam suan palam testati veniam etiamnum orantes rursus in squalentissimos carceres detruduntur . . . . . .

LI. De miserrima tortura cardinalis de Sangro, ac quomodo quaestionibus subiectus tandem pulchro commento auctoris liberatur. Omnia illic horroris plena

LII. De Basilii tormentorum praefecti vita ac moribus truculentissimis. Item de pontificis crudelitate in cardinalem Venetum senio et morbis fractum, qui fidiculis, quoties sustollebatur, orabat: Christus factus est pro nobis obediens etc. . . . . .

LIII. De admonitione fugae quibusdam scriptoribus bullarum torquendis per auctorem significata . . .

LIV. Urbanus in arce Luceriana a Carolo obsessus tandem per quendam Raimundum atque Germanos fortissimos liberatur. . . . . . . . . . .

LV. Quis obsidionis tempore curialium status fuerit ac de abitione auctoris . . . . . . . . . .

LVI. Episcopus Aquilonensis ab Urbano cum cardinalibus captus misereque cruciatus, dum Urbanum fugientem ob membrorum tormentis luxatorum debilitatem sequi non posset, ipso mandante Urbano in itinere mactatur atque insepultus abicitur . .

LVII. Urbanus in Siciliam traiciens Panormi consistens captivos secum abducit, e quibus Angliae cardinalem rebus ac dignitatibus privatum dimittens caeteros miserrima detinet custodia . . . . . . . . 102

LVIII. De genealogia Lupoldi ducis Austriae et de affinitatibus per reges Poloniae, Hungariae, Angliae atque Hispaniae contractis. Eius temporis res gestae obiter referuntur . . . . . . . . . . . . 104

LIX. De morte Caroli regis ac rebus inde secutis . . 107

LX. De liberatione Ottonis ducis eiusque ad Neapolim reditu ac de miserrima quinque cardinalium captivorum caede . . . . . . . . . . . . 
LXI. Duorum e factione Urbani cardinalium ad Clementem defectio . . . . . . . . . . . . 111

LXII. Otto dux foedus cum Apuliae primoribus iniens regnum Siciliae in potestatem redigit. . . . . 111

LXIII. Quid cum Margaretha Caroli regis vidua eiusque filiis exulibus actum sit . . . . . . . . . 112

LXIV. De intercessione matronarum [rectius: Florentinorum] atque nobilium [rectius: aliarum civitatum] pro Margaretha ac pro Caroli cadarere sepeliendo apud Urbanum . . . . . . . . . . . .

LXV. Miles Ottonis a civibus Neapolitanis licenter proditionis vindictam exigens ab Ottone coercetur. 114

LXVI. Principes Germaniae multis rationibus Urbano, ut unioni intendat, persuadere non possunt. . .

.LXVII. De Francisco nepote ob flagitium pulchre dedolato. Item de cardinale Manupelli capto eiusque fratre in exilium acto . . . . . . . . . . . .

LXVIII. Pulcherrima scituque dignissima de pontificum in prostituendis indulgentiis solertia et quam sit hic quaestus multo uberrimus, quales item exitus hic caupones fere sortiantur . . . . . . . . .

LXIX. Urbanus Siciliam profecturus muli lapsu solo graviter illisus, cum illi aes pro militum stipendiis deesset, Romam revertitur, illic paulo post moritur

\section{LIBER SECUNDUS.}

I. De patria, forma ac moribus Clementis VII. deque comitatu Gebennensi in Gallia. Item de paupertate eiusdem ante pontificatum, et quo pacto legationem in Lombardia gesserit . . . . . . . . .

II. De miseranda atque atrocissima civium Cesenatensium nece a militibus Gregorii XI. ad illorum tutelam illic missis peracta . . . . . . .

III. De avaritia Clementis ante pontificatum ac de truculentia tyrannideque Bernabovis Galeacii . .

IV. De Clementis in militares suos nobiles profusa largitione 
V. Venceslai Romanorum ac Hungariae regis ignavia,

Pag. et quo pacto a clericis expeditionis praetextu decimam exegerit . . . . . . . . . . . .

VI. Petrus deThomacellis post Urbani mortem electus Bonifacius IX. appellatur, de forma et moribus eiusdem

VII. De detestanda circa ecclesiasticarum rerum administrationem pontificis avaritia. Item de annatis et fructuum ecclesiasticorum primitiis. . . . .

VIII. De gratiarum, quas vocant, prioritate sordidissime divendita et de dolo a curialibus circa eam rem passim impudentissimeque adhibito. . . . . .

IX. Quo pacto aliae atque aliae insuper artes, doli ac fraudes a sacerdotiorum nuncupatoribus confictae sint. Item de cancellariae regulis aliisque multis eiusdem farinae ad eandemque officinam uti mire conducentia, ita cognitione necessaria . . . . .

$\mathrm{X}$. De dispensationibus Romanis earumque avaritia atque iniquitate, qui curiae praesidebant, omnia stomacho dignissima . . . . . . . . . . .

XI. De sordidissimo Bonifacii in signandis libellis supplicatoriis quaestu . . . . . . . . . . .

XII. Novum commentum Bonifacii ad emulgendas a creaturis suis pecunias illisque pro commodis extollendis spoliandisve . . . . . . . . . . . .

XIII. Bonifacius a matre omnium avarissima non multum degenerans divi Petri auget patrimonium . . . 140

XIV. Idem pontifex aedificiorum struendorum insania. Item de inclementia eiusdem in praelatos. Item de Roberto Palatino Romanorum rege et de pon-

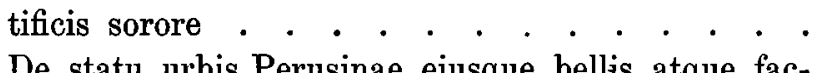

XV. De statu urbis Perusinae eiusque bellis atque factionibus intestinis . . . . . . . . . . .

XVI. Bonifacii iussu Beordus fortissimus Perusinorum praefectus dolo obtruncatur . . . . . . . .

XVII. Ladislaus a Bonifacio rex Hungariae coronatus rebus ecclesiae parum invigilans inglorius ex eodem regno in Siciliam redit . . . . . . . . . . .

XVIII. Sigismundus Hungariae rex totaque Hungaria ob Bonifacii perfidiam contra ecclesiasticos tumultuantur 
XIX. Bonifacius balneandi gratia Puteolas proficiscitur, eius balnei amoenitatem atque salubritatem auctor describens mira in cuiusdam montis vicini meatu subterraneo dicit se vidisse $\cdot$. . . . . . . . 153

XX. Balnei Puteolani efficaciam rursus commendat et de monte, quem vulgus Veneris appellitat. Item de cuiusdam balnei sicci natura . . . . . .

XXI. De ruinis atque vestigiis aedificiorum circa Neapolim et de monumentis Vergilii ibidem . . . .

XXII. Tria milia hominum receptacula subterranea montis circa Neapolim et quaedam alia vetustatem testantia, lectu iucundissima ex sententia Francisci Petrarchae referuntur. Iterum quae de Vergilii magia atque monumentis vulgo dicuntur, an vera vel fabulosa sint, iudicium eiusdem Petrarchae. Item urbs Neapolitana commendatur . . . . . . . . 158

XXIII. Auctor redit ad historiam, quae inter Petri Lunensis nuncios atque Bonifacii acta sint, describens .

XXIV. Moritur Bonifacius, nuncii Petri carceribus mancipantur atque pecunia liberantur . . . . . .

XXV. Quanto pretio Galeatius a Ladislao [rectius: Venceslao] Romanorum rege ducis Mediolani nomen redemerit et de laude Ottonis primi. Item quo pacto Carolus IV. Romanorum rex imperii iura

- dissipaverit, ruinam eiusdem imperii deplorat auctor ibidem . . . . . . . . . . . . . . . 166

XXVI. De quibusdam Scotis pseudoprophetis, quo pacto toti fere Italiae illuserint . . . . . . . . . .

XXVII. Nicolaus Columnensis contra Bonifacium urbem noctu intrans proposito excidit, quibusdam suorum comprehensis atque supplicio affectis. Illic miserum ac crudele facinus eius, qui patrem ac fratrem laqueo suspendit, refertur . . . . . . . . . . .

XXVIII. Jubilaeum visitantes spoliantur. Matronae atque virgines rapiuntur atque abducuntur a latronibus .

XXIX. Cometa apparuit. Guillelmus Gelriae et Joannes Mediolani duces moriuntur. Liguria intestinis seditionibus laborat. Tamberlani victoria contra Bayzetum Turcarum imperatorem . . . . . . 
XXX. Tamberlanus Tartarorum rex Damascum capit atque Pag. diripit. Smyrnae arx omnium munitissima ob arrogantiam praefecti fratrum Hierosolymitarum a Tamberlano destruitur. Eiusdem Tamberlani, qui quingentos Mahometi sacerdotes ad templum confugientes concremaverit, responsum. Item quam vim incantationes Magorum habeant, haud parum multa afferuntur . . . . . . . . . . . . . .

XXXI. De signis atque prodigiis, quae praecesserunt mortem Bonifacii. Item de fortuna ac misero Francisci nepotis Urbani exitu . . . . . . . . . . .

XXXII. An liceat summo pontifici vendere sacerdotia absque simoniae nota sive macula. . . . . . .

XXXIII. De Petri Lunensis alias Benedicti XIII. patria, vita eiusque ante pontificatum gestis, de electione eiusdem. Item de iuramento ab eo ac omnibus cardinalibus praestito. Idem quo pacto postea iusiurandi religionem contempserit. Principes Germaniae in Francofurdensi conventu super schismate consultant, ad Bonifacium nuncios mittunt, quos ille beneficiis atque honoribus fascinat inque suam trahit sententiam . . . . . . . . . . . .

XXXIV. Rursus de funere Bonifacii et factione Guelphorum et Gibellinorum et de electione Innocentii VII .

XXXV. Ladislaus Siciliae rex factionibus intestinis ad urbem illectus coronatur. Tumultus Guelphorum et Gibellinorum narratur. . . . . . . . . . . .

XXXVI. De septemvirorum insolentia in pontificem ac de miseranda caede a nepote pontificis illis inlata. Item de tumultu vulgi in clericos... . . . . .

XXXVII. De conflictu militum regiorum contra Innocentii partes ad pontem S. Angeli et immutatione status ac regiminis urbis Romae . . . . . . . .

XXXVIII. Quo ingenio, dolis atque vafritiis utrique pontifices mutuis literis sese ad unionem exhortando orbi Christiano imposuerint . . . . . . . . . . . . . .

XXXIX. De reditu Innocentii in urbem deque laudibus eiusdem, quas tamen sola ambitione obscuravit ac foedavit . 
XL. Pisae a Florentinis Guelphorum proditione capiunPag. tur. Item quod culpa atque desidia Romanorum regum urbes Italiae ab imperio desciverint ibidem

XLI. Ladislaus rex ob impietatem in clericos ab Innocentio damnatur ac rursus reconciliatur . . . .

XLII. Iterum de pontificum collusione deque eadem re auctoris lamentatio . . . . . . . . . . 202

\section{LIBER TERTIUS.}

I. Quomodo post mortem Innocentii cardinales circa novi pontificis electionem sese habuerint et de iureiurando ab eis adhibito . . . . . . . . . 204

II. Angelus Corario Venetus S. Narci presbyter cardinalis eligitur et Gregorius XII. appellatur . . .

III. Historia tenorem publici instrumenti super iuramento praedicto de verbo ad verbum inserit . .

IV. De litteris Gregorii ad Benedictum super unione transmissis . . . . . . . . . . . . . . 209

V. Benedicti responsio ad litteras suprascriptas . . 211

VI. De nequissima atque dolosa machinatione simulationeque Gregorii . . . . . . . . . . . 214

VII. De potestate pontiticum atque imperatorum, et an imperator in temporalibus subsit pontifici . . . 216

VIII. Gesta ac triumphi Dietherici Bernensis . . . . 219

IX. An papa delinquens debeat et possit ab imperatore corripi et de gestis circa eam rem Ottonis Romanorum imperatoris . . . . . . . . . . . 221

$\mathrm{X}$. An expediat reipublicae Christi pontifices Romanorum temporalia terrarumene dominia possidere atcque tenere . . . . . . . . . . . . . 232

XI. An Romani pontifices iuxta canonum decreta a nemine possint seu debeant iudicari . . . . . 222

XII. De coronatione Gregorii eiusque simulata pietate atque religione . . . . . . . . . . . 226

XIII. Nuncii a Gregorio ad Benedictum super unione ineunda mittuntur, inter quos Antonius de Butrio iurisconsultus . . . . .. . . . . . . . . . 227

XIV. Quomodo Gregorius dominandi captus libidine tergiversatur et locum faciendae unionis causatur. . 
$X V$. Nepos pontificis ac monachus quidam pontifici persuadent, ne unioni intendat . . . . . . . . 230

XVI. Gregorius nepotum ditandorum cura agitatus belli semina atque flagitia suscitat . . . . . . 231

XVII. De aperta recusatione Gregorii super unione facienda et de quaestione ab eodem iureconsultis ea le re proposita . . . . . . . . . . . .

XVIII. De vafro ac nequissimo commento pontificatus retinendi gratia a Gregorio apparato . . . . . . 233

XIX. De discessu Gregorii ab urbe et de his, quare Viterbii super unione agitata fuerunt . . . . 236

$\mathrm{XX}$. Tenor concessionis atque donationis nepotibus pontificiis factae, in qua meras nugas, imo apertissima mendacia, et quo pacto turpitudini fructus honestatis praetendatur, videre licet . . . . . .

XXI. Nepotes pontificis divi Petri haeredes facti amplo ditantur patrimonio. Item quo pacto per varias fraudes unio atque pax Christiana turbata fuerit ab ambitiosissimis praesulibus . . . . . . .

XXII. Quibus artibus Gregorius pecunias corraserit sacramque legerit suppellectilem . . . . . . . 246

XXIII. Duos praestantissimos cardinales recta monentes obaudit Gregorius, novas insuper fraudes, quo diutius retineat imperium, commentus, ubi advertere licet, quam irrequieta sit dominandi libido, et quam difficile imperium semel arreptum dimittatur, ut canis a corio nunquam absterrebitur uncto . .

XXIV. Gregorius contra religionem et suorum cardinalium contradictionem nepotes donare galero instituens multis precibus victus desistit . . . . . . . 255

XXV. Veritatis praedicator Gregorio unionem suadere conatus a nepotibus carceribus mancipatur . . . 257

XXVI. Exemplar seu copia fucatae atque ementitae excusationis motae itemque adhortationis ad unionem Benedicto transmissarum . . . . . . . . . 259

XXVII. Responsio Benedicti ad supradictas excusationes et adhortationes . . . . . . . . . . . . 263 
XXVIII. Tractatus, consultationes atque scripta pro parte utriusque contendentis proposita ad differendam unionem pulchre concinnata . . . . . . . . . 269

XXIX. Litterae Ladislai regis ad Florentinos . . . . 274

XXX. Ex sententia Friderici II. Romani imperatoris urbis ruinam auctor deplorat . . . . . . . . 276

XXXI. Gregorius contra iuramentum praestitum nepotes suos creat cardinales. Ibi haud parum multa de eiusdem pertinacia, quae multorum sanis consilits ac precibus emolliri non potuit . . . . . . . 276

XXXII. Cardinalis Leodiensis viri clarissimi abeuntis bona nepotes pontificis diripiunt . . . . . . . .

XXXIII. Cardinalis passim a papa discedunt eius impias censuras ridentes . . . . . . . . . . .

XXXIV. Litterae cardinalis Leodiensis, quibus, quatenus papae sit oboediendum, significat . . . . . 286

XXXV. Epistola Petri de Luna, quae sale multo Gregorium perfricuit. . . . . . . . . . . . . . . 288

XXXVI. Gregorius concilium ad Adquilegium [rectius Aquileiam] indicit novosque creat cardinales . . . .

XXXVII. De consultatione atque definitione conciliabuli per Benedictum in Aragonia celebrati super schismatis materia, quibus miro modo eiusdem fovetur pertinacia

XXXVIII. Cardinales utriusque factionis sese a pontificibus illusos cernentes Pisis conveniunt, generale concilium indicunt Gregorium citantes. . . . . .

XXXIX. Cardinalis Barensis anno domini 1409 concilii legatus ad Germaniam praelatos ad concilium vocat, de actis eiusdem concilii Pisani. Item quomodo Vormatiensis episcopus una cum aliis quibusdam Ruperti regis Romanorum ad Gregorium legatus cardinalatus honorem ultro oblatum recusat. Antonius nepos cardinalis principes Germaniae Franckfurdiae convenit . . . . . . . . . . . .

$\mathrm{XL}$. Auctor pontificum collusionem atque perversitatem detestatus refert causam eiusdem collusionis, ex sententia divi Pauli divisiones ab ecclesia tollendas esse docet. Quorundam opinionem confutat, ubi etiam non parum multa de significatu verbi Oportet colligit 
XLI. Indicia adventus Antichristi, versiculi in Romanorum degenerationem, de Paulo Ursino . . . . 304

XLII. Digreditur auctor recensens imperium atque potentiam Tartarorum, item gesta, fortunam atque habitum Tamberlani clarissimi imperatoris Tartarorum

XIIII. De ruina fidei ac imperii: lege et plorabis. . . 305

XLIV. Processus ac sententia concilii Pisani antipapas utrosque pontificatibus etc. privans ac haereticos eos schismaticosque declarans . . . . . . .

XLV. De his, quae a depositis taliter pontificibus contra cardinales ac concilium attentata fuerint, et de fuga Angeli Corarii alias Gregorii XII. deficiente crumena molendinoque cessante . . . . . . .

XLVI. Angelus rursus differendi retinendique imperii quaerit suffugium, ubi etiam de causis simulatis inter illustrissimos Rupertum Romanorum ac Ladislaum atque Sigismundum Hungariae Bohemiaeque reges

XLVII. Declaratio eorum, quae capite superiori dicta sunt 312 313

XLVIII. Quo pacto Ladislaus [rectius Sigismundus] ob depositionem Venceslai fratris ab administratione Imperii Romani hostis eiusdem imperii factus sit. .

XLIX. Calliditas atque astutia Angeli in cavendis insidiis sibi a patriarcha Aquilegiensi structis, et quo pacto Paulus cubicularius in easdem inciderit, ac rebus fortunisque destitutus turpiter per Utinensem civitatem deductus fuerit . . . . . . . . .

L. Quo pacto quidem Gregorii palpones miseros exitus invenerunt et de perfidia fratriscelli cuiusdam a Gregorio infestati atıue in reprobum sensum transformati . . . . . . . . . . . . . . .

LI. De Alexandri V. vita ac moribus deque nimia eiusdem in exaudiendis passim precibus facilitate. .

LII. Ludovicus Andegavensis contra Ladislaum Siciliae rex pronunciatur. Rursus de dicti Alexandri indulgentia atque facilitate parum discreta . . .

LIII. De simultate inter Alexandrum V. et Rupertum Romanorum regem. Joannes episcopus Moguntinus creatur natus legatus in sua provincia. Franciscani triumphant. Alexander pie moritur . . 